\title{
TWO DIMENSIONAL MAGNETIC MANIPULATION OF MICRODROPLETS ON A CHIP
}

\author{
U. Lehmann, S. Hadjidj, V.K. Parashar, A. Rida and M.A.M. Gijs \\ Institute of Microelectronics and Microsystems \\ Ecole Polytechnique Fédérale de Lausanne (EPFL), 1015 Lausanne, Switzerland \\ e-mail: ulrike.lehmann@epfl.ch
}

\begin{abstract}
In this paper we describe the two-dimensional (2D) magnetic manipulation of aqueous droplets suspended in silicone oil over the chip surface. The magnetic actuation is based on the force imposed on superparamagnetic microparticles inside the droplets. These can be displaced, merged, mixed and separated by changing the topology of the magnetic field created by a multilayer set of coils. The magnetic manipulation forces are generated on our chip without the use of external moving magnets. Our results demonstrate the potential of the proposed system in droplet-based biomedical analysis methods on a chip.
\end{abstract}

Keywords: Droplet Manipulation, Magnetic Microparticles, Magnetic Actuation, Lab-on-a-chip, Microfluidics

\section{INTRODUCTION}

The handling of small liquid volumes is an important task in miniaturised bio-analytical and biomedical systems where the decreased sample sizes can reduce analysis times and costs. A possible way to scale down sample size and reagent consumption is the use of small droplets. These can be manipulated via electrostatic or magnetic principles $[1,2]$ or by exploiting the properties of electrowetting [3, 4]. The advantages of magnetic actuation for the use in droplet manipulation are the large forces that can be generated and the independence of droplet transport from the dielectric properties of the sample liquid. It has been shown that forces derived from magnetic principles allow the splitting of suspended droplets on a chip. That step was performed by moving an external magnet into the direction of the desired actuation [2].

In our work, we inject superparamagnetic particles into droplets suspended in oil to enable their magnetic actuation. The particles drag the droplet into the direction of an applied field gradient. The force on one magnetic particle in a magnetic induction field $\boldsymbol{B}=\mu_{0} \boldsymbol{H}$ can be expressed as:

$$
F_{m}=(m \cdot \nabla) B
$$

with $\boldsymbol{m}$ the magnetic moment of the particle. The magnetic moment depends on the susceptibility $\chi$, the magnetic volume $V$ of the particle and the field strength $\boldsymbol{H}$ as follows:

$$
\boldsymbol{m}=V \chi \boldsymbol{H}
$$

The actuated particles move inside the droplet until they reach the droplet wall, on which they transfer their actuation force. The strength of the wall is determined by the interfacial tension $\gamma$ between the droplet solution and the surrounding medium. Thus $\gamma$ has a great influence on the displacement properties of the droplet. Another parameter influencing droplet transport is friction $\boldsymbol{F}_{\boldsymbol{R}}$ between the droplet and the chip surface. For successful droplet transport, the friction needs to be kept sufficiently small. The difference between the magnetic force $N \boldsymbol{F}_{\boldsymbol{m}}$ on the $N$ particles in the droplet and the droplet friction $\boldsymbol{F}_{\boldsymbol{R}}$ and the interfacial tension $\gamma$ determine the properties of droplet transport. If the interfacial tension $\gamma$ is smaller than the pressure on the droplet wall exerted by $\boldsymbol{F}=N \boldsymbol{F}_{\boldsymbol{m}}-\boldsymbol{F}_{\boldsymbol{R}}$ the droplet will deform and eventually break; else it will move with the particles.

\section{SYSTEM SETUP}

The main component of our 2D magnetic droplet manipulation system is a four layer Printed Circuit Board (PCB) placed on a soft magnetic sheet with permanent magnets ${ }^{1}$ on the sides. The permanent magnets create a homogeneous static field of $20 \mathrm{mT}$. The homogeneous field is superposed with the changeable field generated by the PCB.

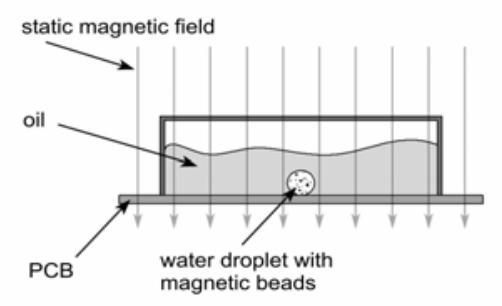

Figure 1: Schematic setup of the magnetic droplet manipulation system.

On the PCB we place an oil reservoir wherein the droplet manipulation is performed (figure 1). The

\footnotetext{
${ }^{1}$ Material: NdFeB
} 
bottom of the $2 \mathrm{~cm} \times 3 \mathrm{~cm}$ large reservoir is formed by a layer of Teflon foil of $12.5 \mu \mathrm{m}$ thickness (DuPont). The Teflon bottom of the reservoir provides a surface with very low friction and a high hydrophobicity. To change the surface properties, in order to increase the friction or wetting angle, the Teflon can be treated with oxygen plasma [5]. The reservoir is filled with silicone oil. The oil is chosen for its suitability in density, viscosity and interfacial tension to allow free floating of the aqueous droplets at the reservoir bottom. Due to the density adjustment of the oil, the droplets can maintain their spherical shape which helps decreasing the friction between droplet and bottom (figure 2).

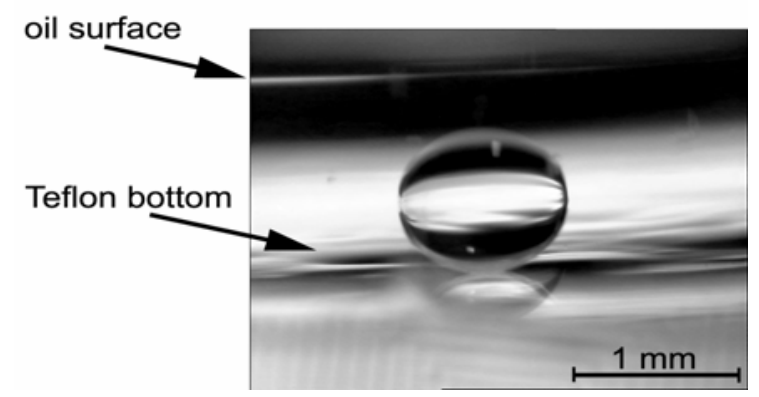

Figure 2: Side view of a suspended droplet on the Teflon surface. The droplet maintains its spherical shape while sitting at the bottom of the reservoir.

As medium for the experiments we chose octamethyltrisiloxane for its density close to water ( $\rho=0.84$ ) and low viscosity ( $\eta=1.2 \mathrm{mPa}$ ) as a basis. In addition, we adjusted the interfacial tension $\gamma$ between the medium and water by adding surfactants. This facilitates the droplet splitting, which highly depends on $\gamma$.

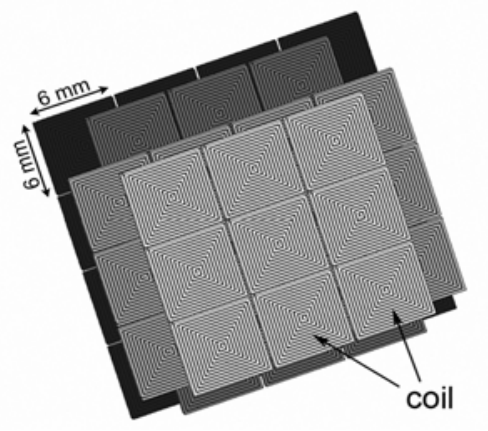

Figure 3: Schematics of the four-layer PCB (explosion view) with coils $\left(6 \times 6 \mathrm{~mm}^{2}\right)$ of 12 windings each; the coils in the different layers partially overlap.

We inject superparamagnetic microparticles into the droplets to provide the means of actuation. Different types and amounts of commercially available magnetic particles were tested in our droplet manipulation system (Table 1).
Table 1: Types of commercially available magnetic particles used in the experiments

\begin{tabular}{|l|l|c|}
\hline \multicolumn{1}{|c|}{ type } & $\varnothing$ (avg.) & material \\
\hline Micromod Nanomag & $250 \mathrm{~nm}$ & magnetite/silica \\
\hline Ademtech Masterbeads & $500 \mathrm{~nm}$ & magnetite/silica \\
\hline Roche (DNA kit) & $6 \mu \mathrm{m}$ & magnetite/silica \\
\hline
\end{tabular}

The magnetic beads inside the droplets on the chip surface are magnetised due to the homogeneous magnetic field of the permanent magnets and form fine pillars inside the droplet. These pillars can be displaced by applying a magnetic field gradient due to changing the topology of the magnetic field. This is achieved via activation of the coils in the different PCB layers. The coils in the different layers partially overlap to as is presented in figure 3.

Depending on the current in each coil, the position of the local magnetic field maxima changes thereby providing a time-dependent field gradient. In addition, the PCB design allows the formation of magnetic channels over the chip surface. These channels are created via the uniform activation of two layers (figure 4). Along the gap between the coils, a chain of local field maxima is formed with confines the superparamagnetic particles along this line without the need for physical barriers. Figure 5 presents the magnetic induction over the coil array as determined by a miniaturised Hall probe, clearly showing the channel-like patterns induced by the actuation of two layers of coils.

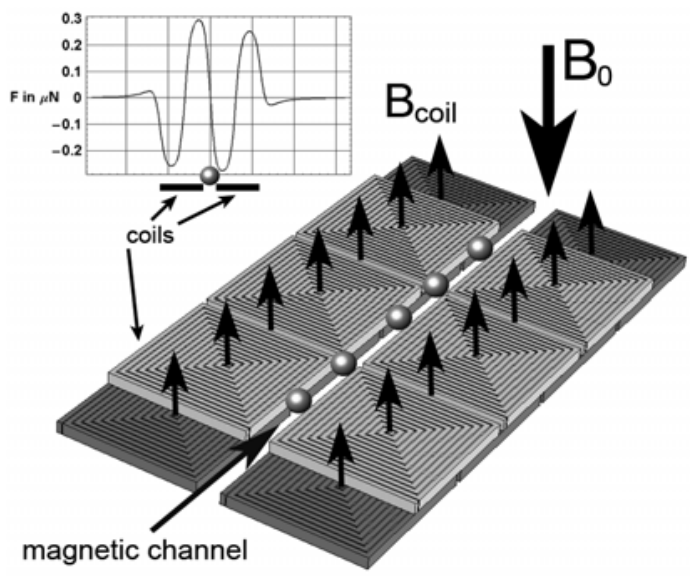

Figure 4: Schematics of the magnetic channel formed by two layers of partially overlapping coils. Inset: analytical simulation of the magnetic force profile transverse to the channel.

The particles that are collected in the channels are transported by a 3-phase activation of the two remaining layers [6]. Local field maxima are created over every third coil, while field minima are introduced over the other coils. Thus the magnetised droplets will 
be dragged into the desired direction along the field gradient.

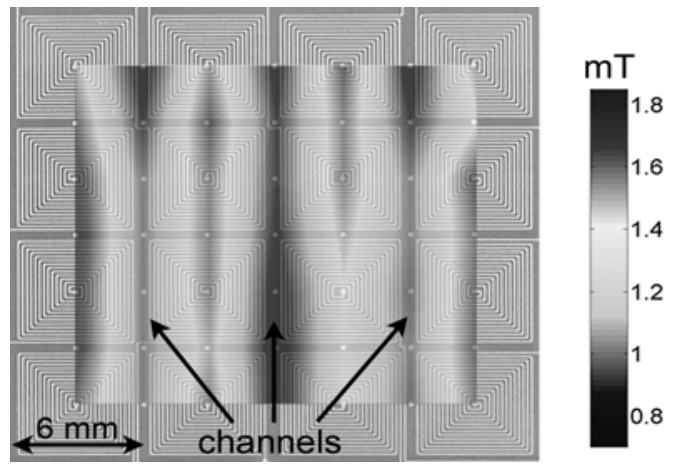

Figure 5: Measurement of the magnetic induction profile over the PCB using two uniformly activated layers of coils.

\section{EXPERIMENTAL RESULTS AND DISCUSSION}

For each particle type, we measure the droplet speed at different particle concentrations in order to determine the obtained force. In parallel, the influence of the particle size on the droplet deformation during transport is examined. For all experiments, the droplet volume is set to $3 \mu \mathrm{l}$ and the droplets are manipulated sequentially on the chip. The droplet displacements are registered via a microscope (Zeiss Stemi V6) with CCD camera. Figure 6 is a sequence of images showing how a droplet can be transported in 2D around a square coil by interchanging the channel-forming and transporting role of layers of coils of the PCB.

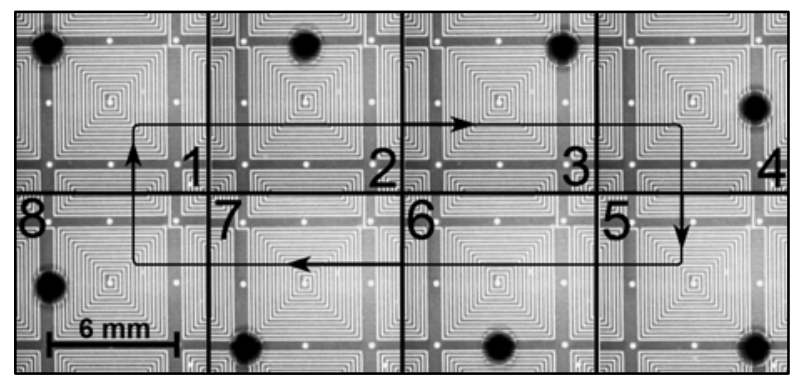

Figure 6: Transport of a $3 \mu \mathrm{l}$ droplet around a square coil on the chip. The magnetic content is $90 \mu \mathrm{g}$ of Micromod beads (diameter $250 \mathrm{~nm}$ ). The droplet speed is about $2 \mathrm{~mm} / \mathrm{s}$.

In addition to the droplet transport, we examine the droplet separation. Here the droplets need to be fixed to a hydrophilic spot on the Teflon foil in order to provide a friction-like counter force against the magnetic pull of the particles. By plasma oxidation of the Teflon, we obtain sufficiently hydrophilic areas to perform the splitting experiments. Since the transport experiments give a first indication of the forces obtainable by the magnetic particles inside the droplets, we can estimate the amount of particles necessary for the droplet splitting.

Figure 7 shows our first separation experiments using the Roche beads, which make a high particle content inside the droplets easily achievable.

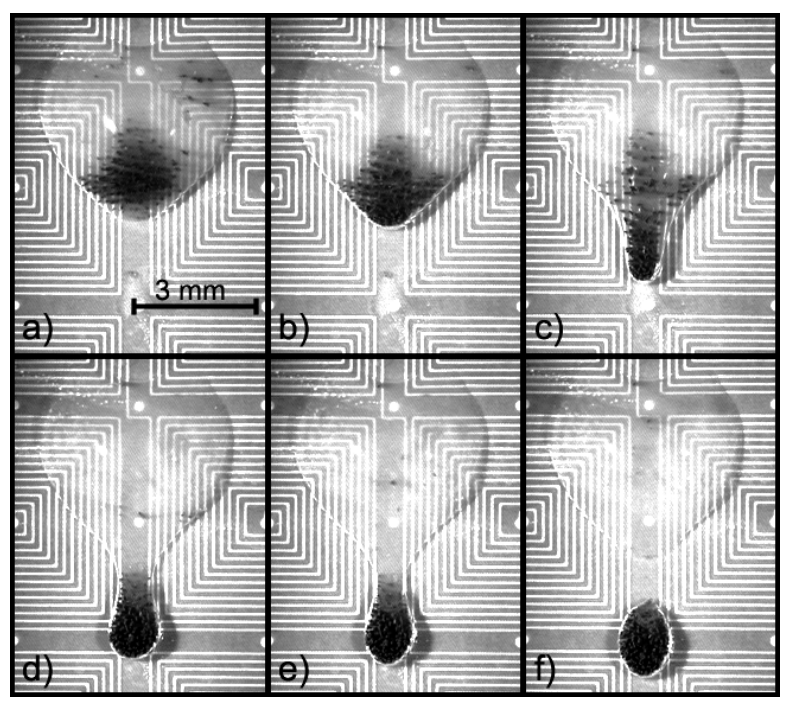

Figure 7: Magnetic bead assisted splitting of an aqueous droplet $(10 \mu \mathrm{l})$. The separated magnetic droplet contains $2 \mu \mathrm{l}$ of magnetic beads (Roche) and liquid. Images (a) - (f) are captured from a film taken during manipulation.

Figure 7 also indicates that we can change the form of the droplets that are fixed on the chip by giving the hydrophilic area a defined shape. The introduction of an extrusion from the usual round droplet shape facilitates the separation, since the energy needed to deform the droplet is decreased. Prior to the droplet separation experiments, there is a more trivial step of droplet merging involved, since this provides an easy way to inject a given amount of magnetic material into a larger droplet.

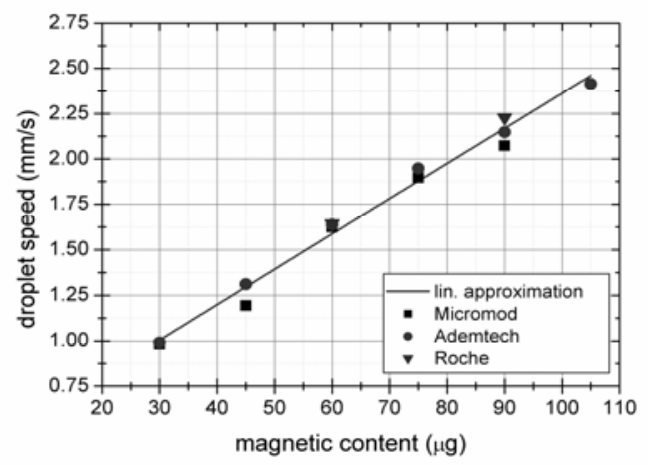

Figure 8: Measurements of the droplet speed for different particle types and concentrations.

Our measurements of the droplet speed for different particle types and different magnetic content show the expected linear behaviour (figure 8). 
Due to the small dimensions and relatively low speed the relation for the viscous drag force

$$
\boldsymbol{F}_{\boldsymbol{d}}=3 \pi \eta D \boldsymbol{v}
$$

can be applied, with $\eta$ the viscosity, $D$ the droplet diameter and $\boldsymbol{v}$ the measured droplet speed. Thus the force generated by the particles inside the droplets can be estimated. Since the friction is expected to be very low the drag force is equal to the magnetic force created by the particles inside the droplet. Figure 9 shows that the forces we can obtain are in the nN-range with a slope of about $1 \mathrm{nN}$ per $\mu$ g. This demonstrates the large forces that can be achieved on our chip.

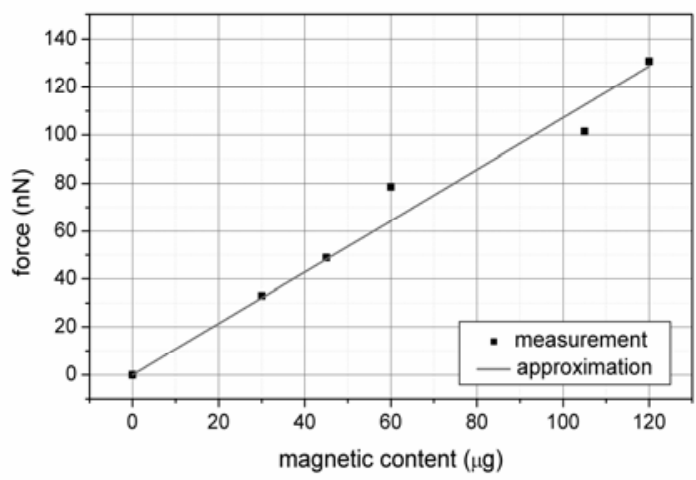

Figure 9: Force created by the magnetic particles inside a $3 \mu \mathrm{l}$ droplet. The force has been derived from the displacement speed of a droplet with Micromod particles in octamethyltrisiloxane ( $\eta=1.2 \mathrm{mPa} \mathrm{s}$ ).

During the droplet transport, we also noted differences in the droplet deformation depending on the type of particle used. For the smallest particles (Micromod), the deformation of the droplet during transport is small. Here the particles are creating a homogeneous force on the droplet wall, since they keep their colloidal state. The larger particles (Ademtech and Roche) lead to a distinct droplet deformation, with a stronger effect for the larger Roche-type beads. In addition, the particles assemble around the field maxima and do not stay in a colloidal suspension. From this observation, we conclude that the larger particles $(>500 \mathrm{~nm})$ are better suitable for droplet splitting since a successful separation is preceded by a deformation of the droplet. In figure 7 , we demonstrate droplet splitting using $2 \mu \mathrm{l}$ of a highly concentrated Roche bead solution (140 mg/ml). In combination with the preceding force measurements, we can estimate the force needed for a successful droplet separation to be about $\mathrm{F}=280 \mathrm{nN}$. Based on this result, we can examine if droplet separation is also possible with smaller beads. Using $5 \mu \mathrm{l}$ of Ademtech beads with a concentration of $50 \mathrm{mg} / \mathrm{ml}$ will create a force of about $250 \mathrm{nN}$ on the droplet wall, which is in the range of the successful separation using the Roche particles. Several tests confirmed a successful separation of a $12 \mu \mathrm{l}$ droplet with this type of beads. However, experiments with a high concentration of Micromod particles proved not to be successful, since the magnetic force could not be sufficiently focussed due to colloidal state of the particles.

\section{CONCLUSION}

We successfully demonstrated the transport, merging and splitting of aqueous droplet on a chip. Our system does not require external moving parts which is a great advantage since this facilitates the fabrication and use of our system. We showed that the field topology created by the PCB can replace physical channels for the transport and manipulation of liquids in droplet shape. By tuning the hydrophobic / hydrophilic surface properties of the oil reservoir, we presented a way to perform droplet splitting on the chip. We also showed that different types of magnetic particles are unequally suited for the manipulation steps. Since the separation appears to be the most crucial step in droplet manipulation, large particles with a diameter $\mathrm{d}>500 \mathrm{~nm}$ are preferable in our droplet manipulation system. Finally, the ability to move, merge, mix, hold and separate droplets on the chip surface opens the way to performing sequential bioanalytical processes on our chip.

\section{Acknowledgements}

We thank Dr. C. Vandevyver for stimulating discussions.

\section{References}

[1] O.D. Velev, Brian G. Prevo and Ketan H. Bhatt, “On-chip manipulation of free droplets," Nature, 426, 515-516 (2003)

[2] M. Shikida, K. Inouchi, H. Honda and K. Sato, "Magnetic handling of droplet in micro chemical analysis system utilizing surface tension and wettability," Tech. Digest, IEEE MEMS 2004, 359362, Maastricht, Netherlands, Jan. 25-29, 2004

[3] S.K. Cho, H. Moon and C.-J. Kim, "Creating, Trans-porting and Merging Liquid Droplets by Electrowetting-Based Actuation for Digital Microfluidic Circuits,” JMEMS, 12(1), 70-80 (2003)

[4] J.A. Schwartz, J.V. Vykoukal and P.R.C. Gascoyne, "Droplet-based chemistry on a programmable micro-chip,” LabChip, 4, 11-17 (2004)

[5] N. Inagaki, K. Narushim and N. Tuchida, "Surface characterization of plasma-modified poly(ethylene terephthalate) film surfaces," Journal of Polymer Science Part B-Polymer Physics 42(20), 3727-3740 (2004).

[6] A. Rida, V. Fernandez, M.A.M. Gijs, “Long Range Transport of magnetic microbeads using simple planar coils placed in a uniform magnetostatic field," $A P L$, 83(12), 2396-2398 (2003) 